\title{
Synthesis and Characterization of Electrospun Poly(vinyl pyrrolidone) (PVP) and Poly(vinyl alcohol) (PVA) Nanofibers with Au Nanoparticles
}

\author{
S. Mishra, ${ }^{*}$ S. P. Ahrenkiel,* V. V. Vodnik,** Z. V. Šaponjić,** and J. M. Nedeljković** \\ * Nanoscience and Nanoengineering Ph.D. Program, South Dakota School of Mines and \\ Technology, Rapid City, SD 57701, USA \\ ** Vinča Institute of Nuclear Sciences, P.O. Box 522, 11001 Belgrade, Serbia
}

Electrospinning is an electrical, jet-based method of fabricating nanofibers that involves the application of a very high electrostatic force on the capillary containing the polymer solution or polymer-melt. The fibers are created by an electrically charged jet of the polymer solution, which can be collected on the surface of a grounded template. The incorporation of metal nanoparticles produces functional nanofibers. Among the noble metal nanoparticles, gold nanoparticles are promising because they have electronic, magnetic, optical and catalytic properties [1].

The solution for electrospinning poly(vinyl pyrrolidone) (PVP) nanofibers was prepared by mixing varying concentrations of $10 \mathrm{~nm} \mathrm{Au}$ nanoparticles-colloidal solution and PVP (average M.W. $1,300,000)$ in isopropyl alcohol (IPA). The electrospinning was carried out at $15 \mathrm{kV}$ to produce nonwoven mats of fibers with diameters ranging from $85 \mathrm{~nm}$ to $1 \mu \mathrm{m}$. Using a Hitachi H-7000 FA TEM, we could confirm the presence of Au nanoparticles within the amorphous PVP matrix (Fig. 1A and 1B). The distribution of Au nanoparticles in the PVP fibers was completely random.

Au/PVA nanocomposite films were prepared by using colloidal solution of Au nanoparticles and poly(vinyl alcohol) (PVA) (average M.W. 72,000) as precursors. A colloidal solution of $0.91 \mathrm{mM}$ Au was prepared [2]. Direct mixing of $1 \mathrm{ml}$ of 5 mass\% aqueous PVA solution with $12 \mathrm{ml}$ of 0.91 $\mathrm{mM} \mathrm{Au}$ colloidal solution lead to formation of stable transparent PVA/Au dispersion. The mixture was placed in a Petri dish and dried in air. After solvent evaporation, a transparent, $4.1 \mathrm{wt} \% \mathrm{Au} / \mathrm{PVA}$ film was obtained. PVA nanofibers with Au nanoparticles were prepared by electrospinning a solution made by dissolving the PVA thin films containing Au nanoparticles, with average diameters ranging from $15 \mathrm{~nm}$ to $30 \mathrm{~nm}$, in $\mathrm{H}_{2} \mathrm{O}$ and IPA, with additional PVA (average M.W. 85,000124,000). The solution was electrospun at $15 \mathrm{kV}$ to produce PVA nanofibers embedded with $\mathrm{Au}$ nanoparticles (Fig. 2A and 2B). Energy-dispersive X-ray spectrometry (EDX) was done to confirm the presence of $\mathrm{Au}$ nanoparticles (Fig. 3). Such nanocomposite fibers are of interest as selfassembled templates for bottom-up fabrication methodologies [3].

\section{References}

[1] J. Bai et al., Mat. Chem. and Phys. 111 (2008) p. 205

[2] K. C. Grabar et al., Anal. Chem. 67 (1995) p. 635-743

[3] C. A. E. Hamlett et al., Tetrahedron 64 (2008) p. 8476

[4] This research was supported by the Department of Energy: "Establishment of the South Dakota Catalysis Group at the University of South Dakota and South Dakota School of Mines and Technology: Advanced Catalyst Materials for Solar Energy Utilization” 

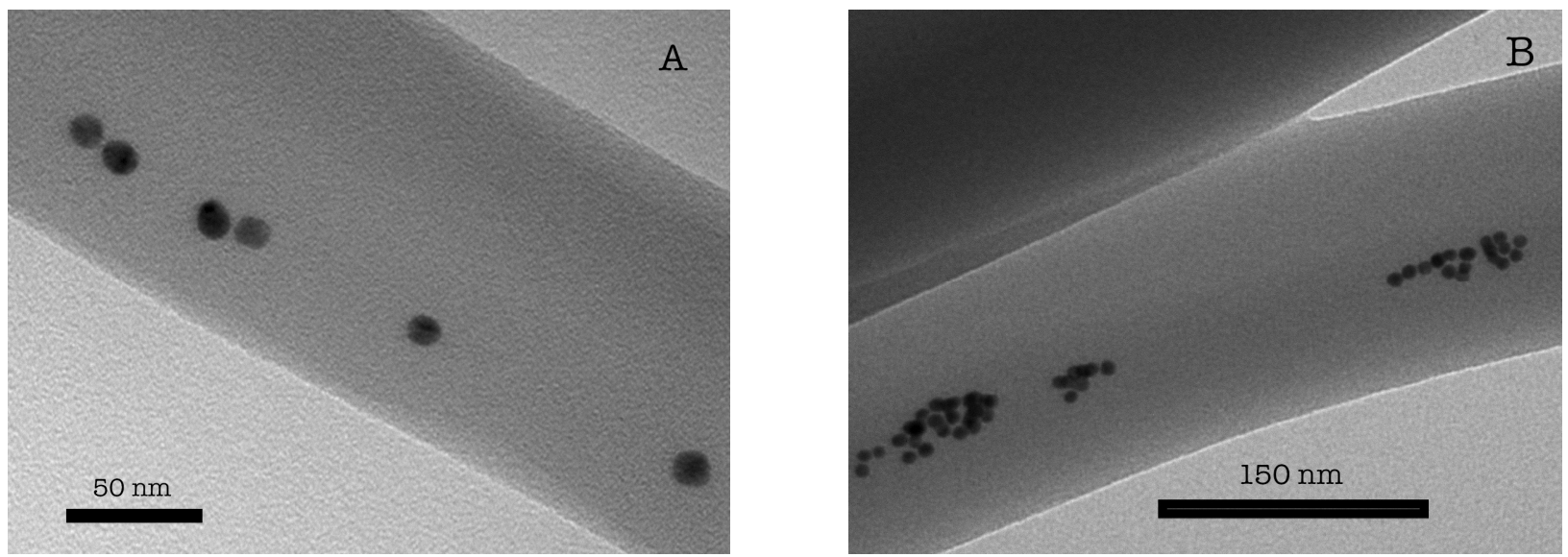

FIG. 1. (A) and (B) TEM images of electrospun PVP nanofibers with Au nanoparticles. The ratio of IPA to Au-colloid was 5. Electrospinning was done at $15 \mathrm{kV}$.
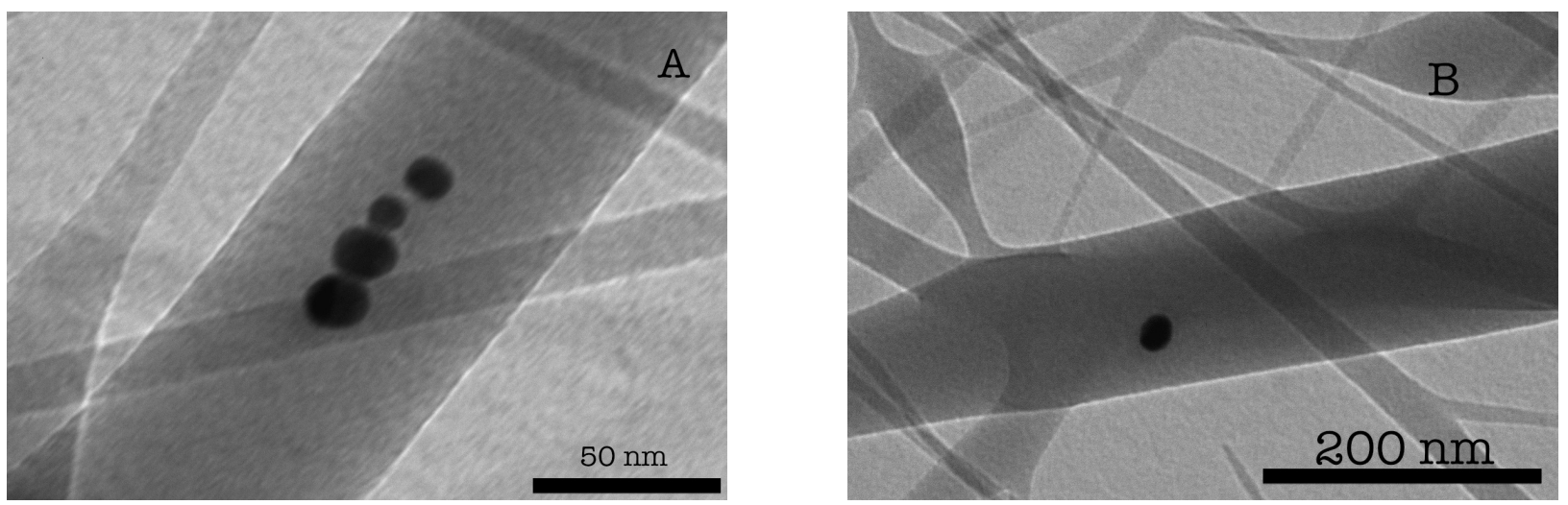

FIG. 2. (A) and (B) TEM images of electrospun PVA nanofibers with Au particles embedded. The solution was prepared by dissolving Au/PVA nanocomposite films in $\mathrm{H}_{2} \mathrm{O}$ and IPA. Electrospinning was done at $15 \mathrm{kV}$.

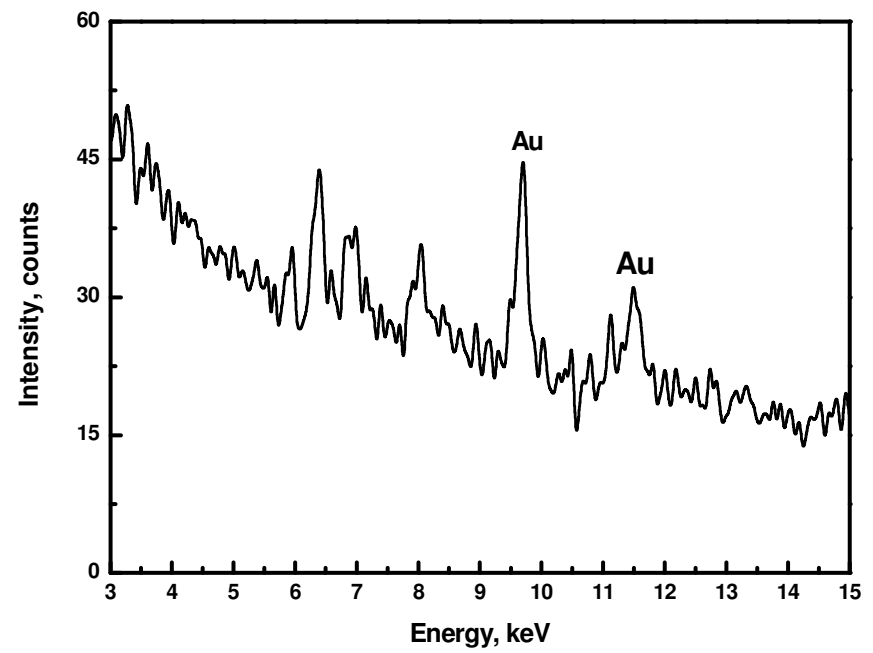

FIG. 3. EDX spectrum taken from Au/PVA electrospun nanofibers showing the presence of Au. 